\title{
OPEN Inspecting the electronic structure and thermoelectric power factor of novel p-type half-Heuslers
}

\author{
Shakeel Ahmad Khandy
}

In line for semiconducting electronic properties, we systematically scrutinize the likely to be grown half-Heusler compounds $X T a Z(X=P d, P t$ and $Z=A l, G a, I n)$ for their stability and thermoelectric properties. The energetically favored $F-43 m$ configuration of XTaZ alloys at equilibrium lattice constant is a promising non-magnetic semiconductor reflected from its total valence electron count $\left(N_{V}=18\right)$ and electronic structure calculations. Alongside mechanical stability, the dynamic stability is guaranteed from lattice vibrations and the phonon studies. The energy gaps of these stable Ta-based materials with $Z=G a$ are estimated to reach as high as $0.46 \mathrm{eV}$ when $X=P d$ and $0.95 \mathrm{eV}$ when $X=P t$; however, this feature is reduced when $Z=A l / I n$ and $X=P d / P t$, respectively. Lattice thermal conductivity calculations are achieved to predict the smallest room temperature value of $K_{L}=33.6 \mathrm{~W} / \mathrm{K}$ (PdTaGa) and 38.0 W/mK (for PtAlGa) among the proposed group of Heusler structures. In the end, we investigated the plausible thermoelectric performance of $X T a Z$ alloys, which announces a comparable difference for the $n$-type and $p$-type doping regions. Among the six alloys, PtTaAl, PtTaGa and PtTaln are predicted to be the most efficient materials where the power factor (PF) elevates up to $\sim 90.5$, $106.7,106.5 \mathrm{~mW} /\left(\mathrm{K}^{2} \mathrm{~m}\right)$, respectively at $900 \mathrm{~K}$; however the lower values are recorded for PdTaAl ( 66.5), PdTaGa ( 76.5) and PdTaln ( 73.4) alloys. While this reading unlocks avenues for additional assessment of this new class of Half Heuslers, the project approach used here is largely appropriate for possible collection of understandings to realize novel stable materials with potential high temperature applications.

Fast-track discovery of new Heusler semiconductor phases from high-throughput computations and advancement in experimental procedures have endured this giant family to trigger new and active research tactics for potential applications ${ }^{1-4}$. The recent breakthroughs of having superconducting effects ${ }^{5}$, high Curie temperatures ${ }^{6}$, topological effects like Weyl or Dirac phenomena ${ }^{7,8}$, spin varying electronic structure ${ }^{9,10}$, skyrmions and giant anomalous Hall effects ${ }^{11,12}$ intensified the new paradigm of research on such materials. With the advent of Heusler alloys, practical applications in the device fabrication of spin injectors or magnetic tunnel junctions have gained momentum because of the efficiency and durability ${ }^{13,14}$. Heusler based thermoelectric materials in general have become the spotlight with the recent discovery of a thin layered Heusler material $\mathrm{Fe}_{2} \mathrm{~V}_{0.8} \mathrm{~W}_{0.2} \mathrm{Al}$ exhibiting a figure of merit (ZT) equal to 5 or $6^{15}$. The same dimensionless parameter $\mathrm{ZT}=\mathrm{S}^{2} \sigma \mathrm{T} / \kappa$, governs the efficiency of a thermoelectric module, where Seebeck coefficient (S), electrical conductivity $(\sigma)$ and thermal conductivity $(\kappa)$. However, $\kappa=\kappa_{\mathrm{e}}+\kappa_{\mathrm{l}}$ is inturn the sum of electronic $\left(\mathrm{k}_{\mathrm{e}}\right)$ and phonon $\left(\mathrm{k}_{\mathrm{l}}\right)$ contribution parts of thermal conductivity ${ }^{16}$. Recently, the artificial layers of $\mathrm{Bi}_{2} \mathrm{Te}_{3}$ and $\mathrm{Sb}_{2} \mathrm{Te}_{3}$ displayed the largest values of $\mathrm{ZT}=2.4$ at room temperature ${ }^{17}$; in $\mathrm{SnSe}$, ZT reached a maximum of $2.6^{18}$, and in p-type PbTe-SrTe, $\mathrm{ZT}=2.5^{19}$. Among Heuslers the $\mathrm{TaFeSb}^{20}$ and $\mathrm{Ti} / \mathrm{Sn}$ doped $\mathrm{NbFeSb}^{21}$ have attained ZT equal to 0.5 and 1.1 experimentally. Kaur et al.reported theoretically the p-type semiconductor TiPdSn with 0.74 at $500 \mathrm{~K}^{22}$ and TaIrSn material with ZT of 0.61 at $900 \mathrm{~K}^{23}$, which are only few to report amid the vast database of thermoelectric Heuslers. However, efforts are being made to find the more efficient Heusler thermoelectrics to have increased competence in converting waste heat to direct electricity.

Half-Heuslers $(\mathrm{HH})$ possess XYZ-type composition, specifically holding non-centrosymmetric C1b cubic structure ${ }^{24}$ within F-43m space group. HH's can be thought as a full-Heusler ( $\left.\mathrm{FH}\right) \mathrm{X}_{2} \mathrm{YZ}$ structure in which D $(0.75,0.75,0.75)$ site is empty and the other three sites, $\mathrm{A}(0,0,0), \mathrm{B}(0.25,0.25,0.25)$ and $\mathrm{C}(0.5,0.5,0.5)$ are occupied by Z, Y and X atoms, respectively in a unit cell. The potential feature of electronic structure of such compounds range from half-metallicity ${ }^{25}$ to semiconductor or semimetallic to topological behavior ${ }^{26,27}$. In this article, we examine six half-Heusler compounds with chemical formula XTaZ, where X is (Pd or Pt) atom and Z 


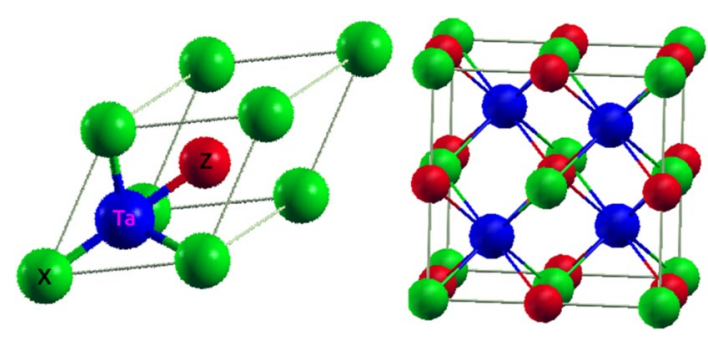

Figure 1. Primitive cell of XTaZ molecule and lattice structure in F-43m configuration. Green balls represent $\mathrm{X}(\mathrm{Pd} / \mathrm{Pt})$, Blue represent Ta atoms and red spheres represent $\mathrm{Z}(\mathrm{Al} / \mathrm{Ga} / \mathrm{In})$ atoms, respectively.

is a group IIIA ( $\mathrm{Al}, \mathrm{Ga}$ or In) element. The primitive cell of MgAgAs type XTaZ HH's alongside the occupation sites of individual atoms is depicted in Fig. 1. The total sum of valence electrons in all the six materials is 18, which significances the exhibition of nonmagnetic and semiconducting nature of all these materials. The semiconductor nonmagnets of XYZ kind have been intensively studied, but no theoretical or experimental work has been reported about the present set of materials till date. Since, the electronic composition of this class is mainly $4 \mathrm{~d} / 5 \mathrm{~d}$ electrons, therefore the electrical conductivity is expected to be larger in comparison to $3 \mathrm{~d} \mathrm{HH}$ systems. This also adds to the feature of heavier mases of constituent elements, which in turn is expected to decrease the lattice thermal conductivity. The main objective is to explore the equilibrium electronic structure of these transition-metal atom based Heuslers for the possible thermoelectric applications. Thus, we first time make use of density functional perturbation theory (DFPT) calculations to determine the stable crystal structure via phonon dispersion. The electronic structure and thermoelectric properties are computed by density functional theory (DFT) and Boltzmann transport theory. To predict the overall physical (structural, electronic, mechanical, phonon) and thermoelectric properties, the paper is organized as follows.

\section{Computational methodology}

The XTaZ (X = Pd, Pt and Z=Al, Ga, In) half-Heusler alloys are proposed to be crystallized in cubic F- $43 \mathrm{~m}$ structure. First, we perform geometry optimization and proper structural relaxation by fixing the volume relaxation of all these 6 materials. Monkhorst-Pack scheme for k-mesh size of $5 \times 5 \times 5$ within an energy cutoff of 450 Ry is employed within QUANTUM ESPRESSO package $\mathrm{e}^{28}$. The kinetic energy cutoff is fixed at $45 \mathrm{Ry}$ for the plane-wave expansion of the electronic wave functions. We choose the Pedrew Burke Ernzerhof (PBE-GGA) correlation functional approximation ${ }^{29}$ with Rappe Rabbe Kaxiras Joannopoulos (ultrasoft) pseudopotential ${ }^{30}$. Keeping the convergence threshold of $10^{-6} \mathrm{Ry}$, SCF calculations are done within a cold smearing under the Methfessel-Paxton scheme set at $0.001 \mathrm{Ry}$. For phonon calculations, both DFT and DFPT simulations on a $5 \times 5 \times 5 \mathrm{q}$-mesh with a threshold of $10^{-12}$ are run for self-consistency. Boltztrap code is employed to calculate the transport properties ${ }^{31}$, but it furnishes the $\sigma$ and $\kappa_{\mathrm{e}}$ in terms of $\tau$. So, the coefficients are described by assuming the electron relaxation time of NiTiSn $\left(\tau=1.5 \times 10^{-14} \mathrm{~s}\right)^{32,33}$ and solving the electronic Boltzmann equation within this approximation. A large k-mesh of $50 \times 50 \times 50$ was used for transport calculations. Slacks model is used to evaluate the lattice thermal conductivity of the present alloys ${ }^{34-36}$.

\section{Results and discussion}

Structure, phonon dispersion and stability. Chemically, it can be said that the group VB element Ta with high-lying atomic orbitals (5d orbitals) easily transfers the charge to the IIIA element (Al, Ga or In) in the present set of materials. However, d-filled atoms $(\mathrm{Pd} / \mathrm{Pt})$ preferably support the chemical bonding in an XTaZ molecule. The proposed XTaZ compounds are optimized in the conventional F- $43 \mathrm{~m}$ structure and the relaxed lattice parameters along with semiconducting energy gaps are listed in Table 1 . In the nearest neighbor coordination, each $\mathrm{X}$ and $\mathrm{Z}$ atom forms $\mathrm{XTa}_{4}$ and $\mathrm{ZTa}_{4}$ tetrahedral structure, respectively. It is noteworthy to mention here that the interchanging of $\mathrm{X}$ and $\mathrm{Y}$ positions as $\mathrm{XYZ}$ and $\mathrm{YXZ}$ in actual crystal are crucial for electronic structure as they may exhibit semiconducting, metallic, or semi-metallic properties. We optimized both XTaZ and TaXZ structures and select the energetically most stable structure. The cohesive energies are of the order of 7.7 eV (PtTaAl), $5.9 \mathrm{eV}$ (PtTaGa) and $4.9 \mathrm{eV}$ (PtTaIn), 10.6 eV (PdTaAl), $5.5 \mathrm{eV}$ (PdTaGa), 9.5 eV (PdTaIn). This energy predicts the phase stability and the bond strengths binding the respective atoms. However, the formation energies for Pt based alloys in order are $-2.04,-2.85,-1.08, \mathrm{eV}$ and Pd based alloys are $0.81,0.95,0.27 \mathrm{eV}$, respectively. Since, the negative values of PtTaX alloys confirms the likeliness of their experimental synthesis but, the PdTaX group is unlikely metastable in nature due to small positive energies. Therefore, a novel Heusler material series is proposed in this report.

The phonon dispersions are calculated to define the vibrational stability of the XTaZ alloys. The positive only phonon frequencies seen in Fig. 2 reflect the stable nature of these materials. Thus, XTaZ alloys are determined to be stable and to the best of our knowledge, these are not been reported in any database yet. Among the nine branches, low-lying three branches are acoustic (lower frequencies) and the six above in pairs of three each are optical modes (higher frequencies). Three degenerate regions are observed at $\Gamma$ point, one acoustic and two optical regions. In all three regions, the transverse modes are doubly degenerate. The maximum acoustic phonon frequency of PdTaAl is $147.62 \mathrm{~cm}^{-1}$, PdTaGa is $135.24 \mathrm{~cm}^{-1}$ and PdTaIn is $148.75 \mathrm{~cm}^{-1}$ while as for PtTaAl, PtTaGa and PtTaIn alloys, the corresponding values are $151.8,143.52$ and $146.62 \mathrm{~cm}^{-1}$, respectively. The experimental 


\begin{tabular}{|l|r|r|r|r|r|r|}
\hline Parameter & PdTaAl & PdTaGa & PdTaIn & PtTaAl & PtTaGa & PtTaIn \\
\hline Lattice constant $\left(\mathrm{a}_{0}\right)$ & 6.03 & 6.02 & 6.24 & 6.06 & 6.05 & 6.26 \\
\hline Band gap $(\mathrm{eV})$ & 0.31 & 0.46 & 0.33 & 0.56 & 0.95 & 0.92 \\
\hline Lattice constants & & & & & & \\
\hline $\mathrm{C}_{11}$ & 214.56 & 203.51 & 215.30 & 256.22 & 145.38 & 227.88 \\
\hline $\mathrm{C}_{12}$ & 124.30 & 136.09 & 114.97 & 136.00 & 237.98 & 118.57 \\
\hline $\mathrm{C}_{44}$ & 83.69 & 85.36 & 69.47 & 96.90 & 95.26 & 76.40 \\
\hline Bulk modulus $(\mathrm{B})$ & 154.3 & 158.56 & 148.42 & 176.07 & 176.24 & 155.00 \\
\hline Young's modulus $(\mathrm{Y})$ & 171.70 & 156.87 & 160.90 & 208.44 & 188.43 & 175.25 \\
\hline Shear modulus $(\mathrm{G})$ & 65.32 & 58.81 & 60.98 & 80.01 & 71.31 & 66.81 \\
\hline Poisson's ratio $(\mathrm{v})$ & 0.31 & 0.33 & 0.31 & 0.30 & 0.32 & 0.31 \\
\hline Pughs ratio $(\mathrm{B} / \mathrm{G})$ & 2.47 & 2.69 & 2.46 & 2.14 & 2.66 & 2.43 \\
\hline Cauchy pressure $\left(\mathrm{C}_{12}-\mathrm{C}_{44}\right)$ & 40.61 & 50.73 & 45.50 & 34.66 & 57.43 & 51.91 \\
\hline Compressional velocity $\left(v_{l}\right)$ & 5045.35 & 4669.12 & 4543.18 & 4848.44 & 4508.83 & 4288.63 \\
\hline Sound velocity $\left(v_{s}\right)$ & 2624.02 & 2326.02 & 2340.71 & 2579.14 & 2311.50 & 2243.65 \\
\hline Average sound velocity $\left(v_{m}\right)$ & 2917.89 & 2575.43 & 2616.20 & 2870.34 & 2567.46 & 2504.85 \\
\hline Debye temperature $\left(\theta_{\mathrm{D}}\right)$ & 329.43 & 291.61 & 286.96 & 322.87 & 289.10 & 272.630 \\
\hline
\end{tabular}

Table 1. Lattice constants (in $\AA$ ), energy gaps (in eV) elastic constants and mechanical parameters (in GPa), Debye temperature (in K), compressional velocity (in $\mathrm{m} / \mathrm{s}$ ), sound velocity (in $\mathrm{m} / \mathrm{s}$ ) and average sound velocity (in $\mathrm{m} / \mathrm{s}$ ) as calculated for XTaZ alloys.
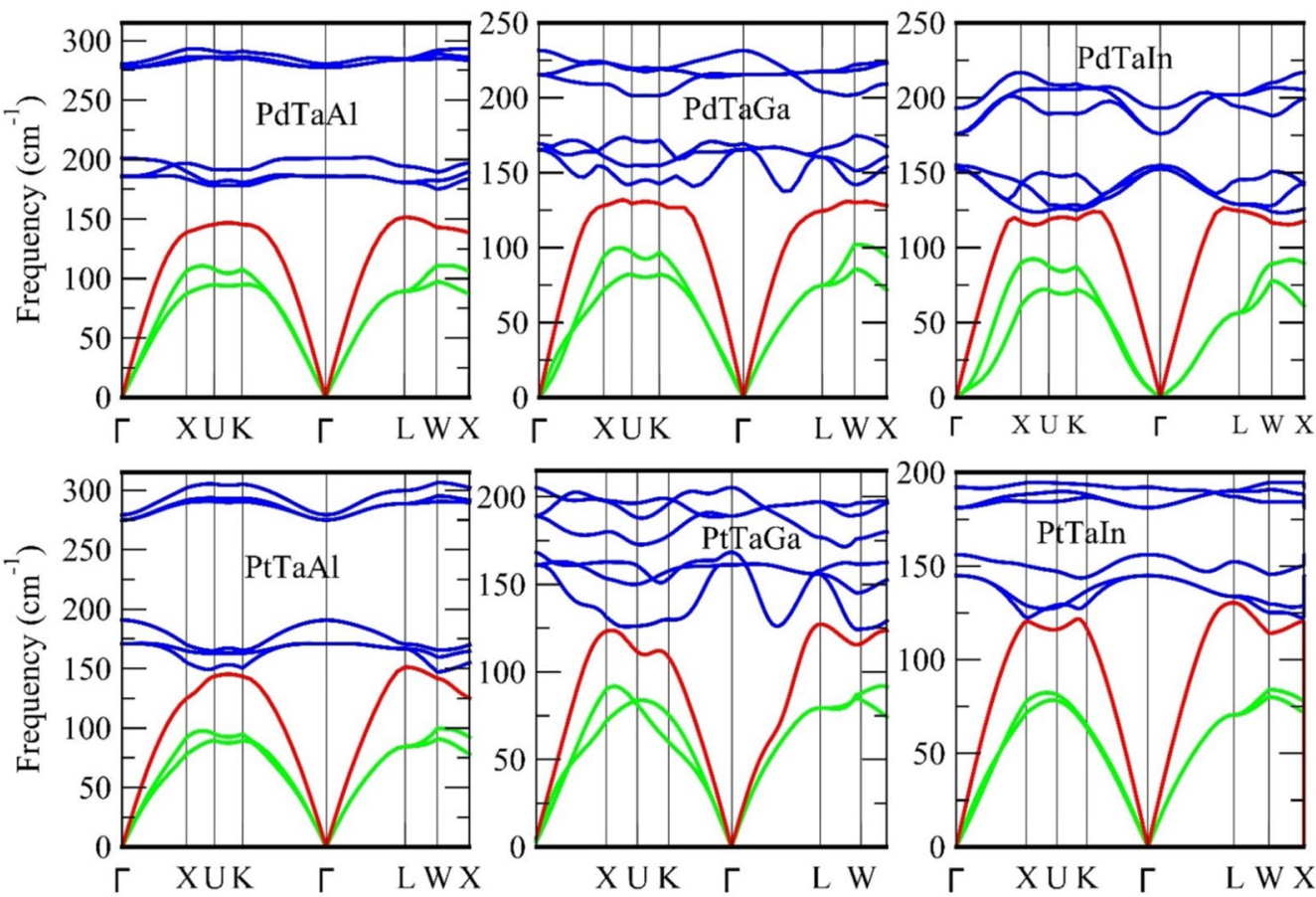

Figure 2. Phonon band structure of XTaZ alloys with no negative frequencies. Two green lines represent transverse acoustic (TA), red line indicates longitudinal acoustic (LA) and blue lines are six optical (O) modes.

thermoelectric material, NbFeSb alloy exhibits the maximum acoustic phonon at $\sim 193.20 \mathrm{~cm}^{-121,37}$. Acoustic modes in the present materials are located below $\sim 150 \mathrm{~cm}^{-1}$ and the optic modes with small gap lie above that range. The optical modes largely being should contribute little towards the heat conduction. The acoustic phonon population is directly linked to the thermal conductivity at low temperature and its small value proves fruitful for less phonon scattering. Because, their maximum velocities majorly decide the lattice thermal conductivity ${ }^{38}$. Therefore, we can presume that the present materials will show less thermal conductivity in comparison to latter. The small gap or maximum overlap between acoustic (LA) and optical (TO) branches is observed which reduces the phonon scattering. In addition, it can be inferred that PtTaGa and PdTaGa may exhibit small conductivity in transport mechanism, which is verified in thermoelectric properties section. Also, the maximum optical mode 
frequency for all these materials is lower than $\mathrm{ZrCoSb}\left(285 \mathrm{~cm}^{-1}\right)^{39}$, which implies the bonding is comparable to that of $\mathrm{ZrCoSb}^{40,41}$. This typical behavior plays a substantial role in deciding the transport properties of the $\mathrm{XTaZ}$ alloys.

Elastic constants and mechanical properties. Keeping in view the structural stability via phonon dynamics and the mechanical standards of the present systems is discussed here. Voigt-Reuss-Hill (VRH) approximation is used ${ }^{42-44}$ for the elastic and mechanical properties analysis, and the subsequent equations are given below $^{45-47}$.

$$
\begin{gathered}
B_{V}=B_{G}=B=\frac{\left(C_{11}+2 C_{12}\right)}{3} \\
G_{V}=\frac{\left(C_{11}-C_{12}+3 C_{44}\right)}{5} ; \quad G_{R}=\frac{5\left(C_{11}-C_{12}\right) C_{44}}{4 C_{44}+3\left(C_{11}-C_{12}\right)} ; \quad G=\frac{G_{V}+G_{R}}{2} \\
\mathrm{Y}=\frac{9 B G}{3 B+G} ; \quad v=\frac{3 B-Y}{6 B} ; \quad A=\frac{2 C_{44}}{\left(C_{11}-C_{12}\right)}
\end{gathered}
$$

B, S, Y are bulk, Shear and Young's modulus, respectively; A is the Zener anisotropy factor, $v$ is is considered as Poisson's ratio. Later, the expedition of Debye temperature $\left(\theta_{D}\right)$ is enlisted from the combinations of compressional velocity $\left(v_{l}\right)$, sound velocity $\left(v_{s}\right)$ and average sound velocity $\left(v_{m}\right)$ as below; ${ }^{48,49}$

$$
\begin{gathered}
v_{s}=\sqrt{\frac{G}{\rho}} \text { and } v_{l}=B=\sqrt{\frac{(3 B+4 G)}{3 \rho}} \\
v_{m}=\left(\frac{1}{3}\right)^{\frac{1}{3}}\left(\frac{2}{v_{s}^{3}}+\frac{1}{v_{l}^{3}}\right)^{-\frac{1}{3}} \\
\theta_{D}=\frac{\hbar}{k_{B}}\left(\frac{3 n}{4 \pi}\left(\frac{\rho N_{A}}{M}\right)\right)^{\frac{1}{3}} v_{m}
\end{gathered}
$$

where $\hbar, n, k_{B}, N_{A}, M, \rho$, are the reduced Planck's constant, number of atoms per formula unit, Boltzmann's constant, Avogadro's number, atomic mass, and density of unit the cell, respectively.

The mechanical stability is one among the concerned factors for choosing a material for desired applications. A material needs to be mechanically stable and practically feasible to be used in a thermoelectric module. Using the volume and energy conserving tetrahedral and rhombohedral distortions across the cubic structure, the various stability conditions taken into consideration are $\mathrm{C}_{11}+2 \mathrm{C}_{12}>0, \mathrm{C}_{11}-\mathrm{C}_{12}>0, \mathrm{C}_{44}>0^{50}$. The various mechanical parameters listed in Table 1 are calculated from elastic constants using the relations mentioned in methodology section. The calculated Bulk, Young's, and shear moduli of the present alloys increase first from Al to Ga and then decrease from Ga to In. Poisson's ratio $(\boldsymbol{v})$ hints the existence of central forces because $\boldsymbol{v}>0.25<0.50$ for all the materials under study, while as $0.25 \leq \boldsymbol{v} \geq 0.50$ classifies the non-central nature of bonding forces in a solid $^{51}$. B/G (Pugh's ratio) as well as accompanied by Cauchy pressure determine the ductile nature of all these Heuslers $^{52,53}$. Using the data sets of various mechanical parameters, we predicted the Debye Temperature $\left(\boldsymbol{\theta}_{\mathrm{D}}\right)$ of 329.43 K (PdTaAl), 291.61 K (PdTaGa), 286.96 K (PdTaIn), 323.58 K (PtTaAl), 278.66 K (PtTaGa), 278.08 K (PtTaIn), respectively.

Electronic structure and density of states. The electronic structure of XTaZ alloys are investigated using the optimized lattice constants. All the three atoms in the present set of $\mathrm{HH}$ alloys exhibit $\mathrm{T}_{\mathrm{d}}$ symmetry in their first coordination sphere and the octahedral symmetry in the next. Here we focus on the band structure along the high symmetry points as shown in Fig. 3. All these materials present a direct p-type band gap at $\mathrm{X}$-point of the Brillion zone. PdTaAl, PdTaGa and PdTaIn show a gap of $0.31 \mathrm{eV}, 0.46 \mathrm{eV}$ and $0.33 \mathrm{eV}$, respectively; while as PtTaAl, PtTaGa and PtTaIn display larger gaps of $0.56 \mathrm{eV}, 0.95 \mathrm{eV}$ and $0.92 \mathrm{eV}$, respectively. In both these cases, this gap increases from $\mathrm{Al}$ to $\mathrm{Ga}$ but decreases again from $\mathrm{Ga}$ to In. This can be attributed to the addition of 5d-orbitals in In-based alloys, which increases the local effects due to extremely large size than that of the 3d-orbitals of Al-type materials. The top valence band exhibits flatness or band convergence, which is said to be favorable for large Seebeck power of these materials. Since, the flatness increases from Al to Ga therefore the Seebeck coefficient is supposed to improve accordingly due to amplified effective mass.

The possible understanding of the semiconducting gap originates from the symmetry and position of constituent atoms, which thereby decide the occupation of bands around the Fermi level (EF). Total and partial density of states (TDOS, PDOS) presented in Fig. 4 are calculated to infer the strength of hybridization, occupation, and contribution from orbital states of individual atoms. XTaZ alloys display the semiconductor type band structure because of the generalized magic number- 18 VEC rule ${ }^{54,55}$ which exhibits the 9 hybridized valence bands to fully compensate the spin effects in such systems. Here, we see the $d$-states of $\mathrm{X}$ and Ta atoms are mostly populating near the EF with an admixture of $p-Z$ states. The orbital occupancies of individual atoms mix according to the octahedral $d$ - $d$ hybridization explained in ref. ${ }^{56,57}$. While interpreting the $p$-orbitals of $Z$ atoms (Fig. $4 \mathrm{a}-\mathrm{f}$ ), it 


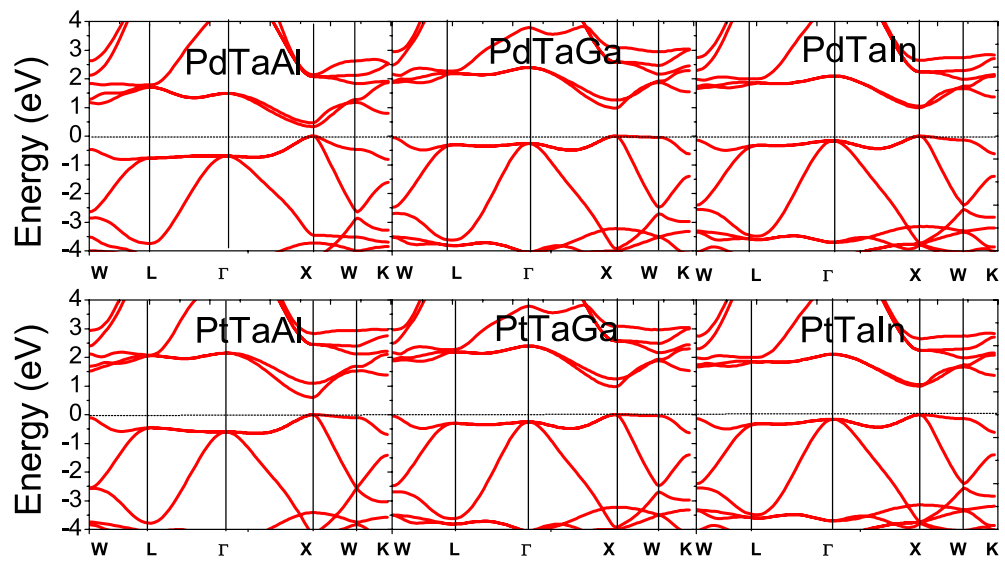

Figure 3. Electronic structure of XTaZ alloys with Fermi level set at zero.
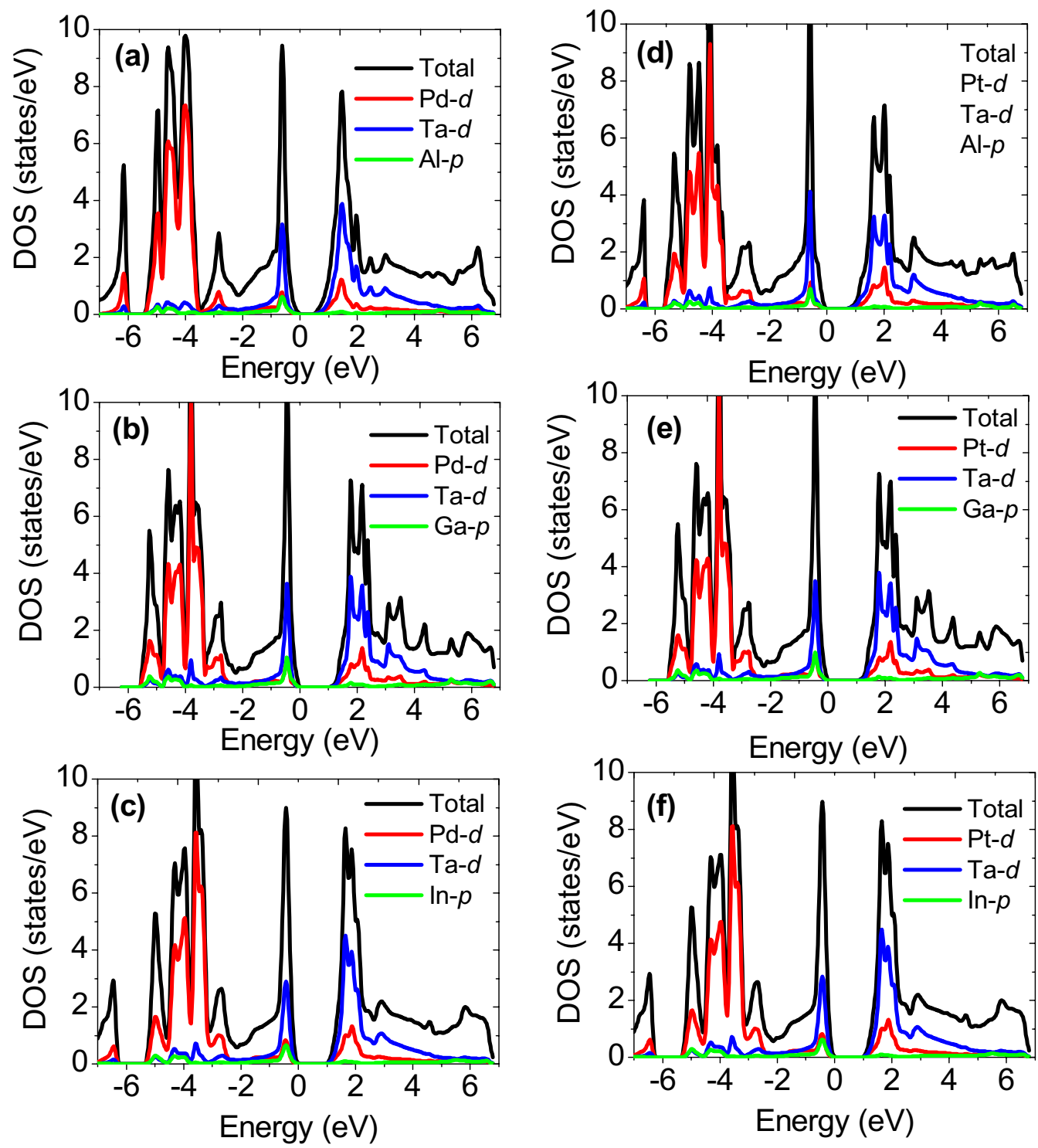

Figure 4. Projected (pDOS) density of states of XTaZ compounds. 


\begin{tabular}{|l|l|l|l|l|l|l|c|}
\hline Parameter & Temp & PdTaAl & PdTaGa & PdTaIn & PtTaAl & PtTaGa & PtTaIn \\
\hline \multirow{2}{*}{$\mathrm{S}(\mathrm{mV} / \mathrm{K})$} & \multirow{3}{*}{$300 \mathrm{~K}$} & 1.5 & 3.5 & 2.8 & 4.0 & 6.0 & 5.0 \\
\cline { 3 - 8 } & & 11.0 & 13.5 & 12.6 & 33.5 & 40.0 & 23.4 \\
\hline \multirow{2}{*}{$\mathrm{PF}\left(\mathrm{mW} / \mathrm{K}^{2}\right)$} & \multirow{2}{*}{$900 \mathrm{~K}$} & 6.0 & 8.5 & 6.5 & 11.0 & 17.0 & 16.5 \\
\cline { 3 - 8 } $\mathrm{S}(\mathrm{mV} / \mathrm{K})$ & 66.5 & 76.5 & 74.4 & 90.5 & 106.7 & 106.5 \\
\hline $\mathrm{PF}\left(\mathrm{mW} / \mathrm{K}^{2}\right)$ & & & &
\end{tabular}

Table 2. Approximate values of Seebeck coefficient (S) and Powerfactor (PF) of XTaZ alloys calculated at $300 \mathrm{~K}$ and $900 \mathrm{~K}$ temperature range.
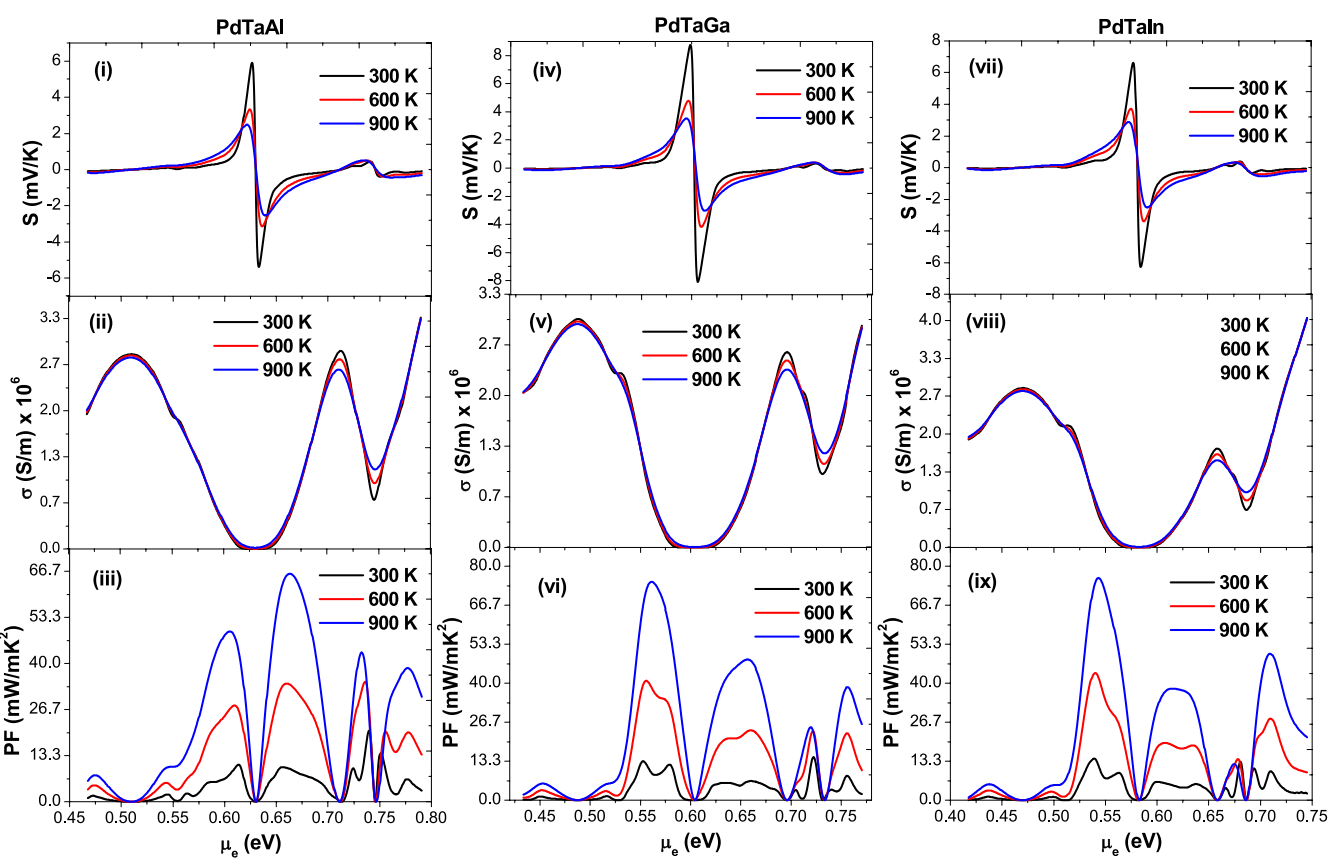

Figure 5. Transport coefficients of $\operatorname{PdTaX}(X=A l, G a, I n)$ alloys as a function of chemical potential: (i, iv, vii) Seebeck coefficient, (iii, v, viii) Electrical conductivity and (iii, vi, ix) Powerfactor.

is observed that they occupy the low-lying bands over the energy range of 0-0.9 eV. However, the $d$-bands of $\mathrm{Pd}, \mathrm{Pt}$ and Ta span from 1 to $4 \mathrm{eV}$ in case of PdTaZ and 2-4 eV in PtTaZ alloys depicting their occupation and strength of hybridization. The two sets of bonding and antibonding orbitals formed from the split of $d$-bands in XTaZ alloys (each set from triple degenerate $(d x y, d x z$ and $d y z)$ orbitals and double degenerate $d x^{2}-y^{2}$ and $d z^{2}$ orbitals near the Fermi level) are responsible for the creation of band gap. Thus, a decisive new group of $\mathrm{HH}$ alloys is supposed to be displaying semiconducting properties.

Thermoelectric coefficients. In Table 2, the Seebeck coefficient (S) and power factors (PF) are listed at $300 \mathrm{~K}$ and $900 \mathrm{~K}$ temperatures. At room temperature, the corresponding values of Seebeck coefficient are in the range of $1-3.5 \mathrm{mV} / \mathrm{K}$ in Pd-based alloys and $4-6 \mathrm{mV} / \mathrm{K}$ in Pt based alloys but interestingly higher temperature fluctuations reduce the magnitude by 5 times (see Figs. 5, 6). This type of behavior is true, mutatis mutandis for electrical conductivity $(\sigma)$ against the same temperature gradient, even though the magnitude fluctuates within the smaller window of $2-3 \times 10^{-6} \mathrm{~S} / \mathrm{m}$. It can be argued that the $\sigma$ first increases as expected w.r.t $\mathbf{T}$ in the band gap region and then decreases away from it on both sides when hole or electron concentration magnifies (see the case of PdTaGa in Fig. 7). This is purely a semiconductor behavior when doped accordingly with n-type or p-type impurities. We also calculate the Powerfactor (PF) of the proposed alloys within the constant relaxation time approximation (see "Methods" for computational details). The large PF in these alloys is expected from the valley degeneracy due to location of CBM and VBM at low symmetry points and the d-orbital contributed high DOS near the Fermi level at the bottom of the conduction band and the top of the valence band seen in Figs. 3 and 4. Subsequently, the flat-and-dispersive band appearances (due to $d$-states) visualized near the Fermi level are in line with our expectations, which increases the PFs from $\sim 10 \mathrm{~mW} / \mathrm{mK}^{2}$ for PdTaAl to $\sim 13.5 \mathrm{~mW} / \mathrm{mK}^{2}$ for PdTaGa and up to 23-44 mW/mK $\mathrm{m}^{2}$ for PtTaIn, PtTaAl and PtTaGa at $300 \mathrm{~K}$ (Figs. 5, 6, and Table 2). Similar type of increase in PF has been attributed to Fe-eg flat-and-dispersive bands in $\mathrm{Fe}_{2} \mathrm{YZ}_{\text {alloys }}{ }^{58}$. However, the present set of novel compounds unveil the massive upsurge in PFs from $\sim 66$ to $107 \mathrm{~mW} / \mathrm{mK}^{2}$ at $900 \mathrm{~K}$. In all Pd and Ptbased compounds exhibiting better TE properties at higher temperatures, the PF rests on substantial magnitude (i.e. $95 \%$ peak) in a comparable and reasonably wide range of carrier concentrations $\left(\mathrm{n} \approx 1 \times 10^{20}-2 \times 10^{21} \mathrm{~cm}^{-3}\right)$ 

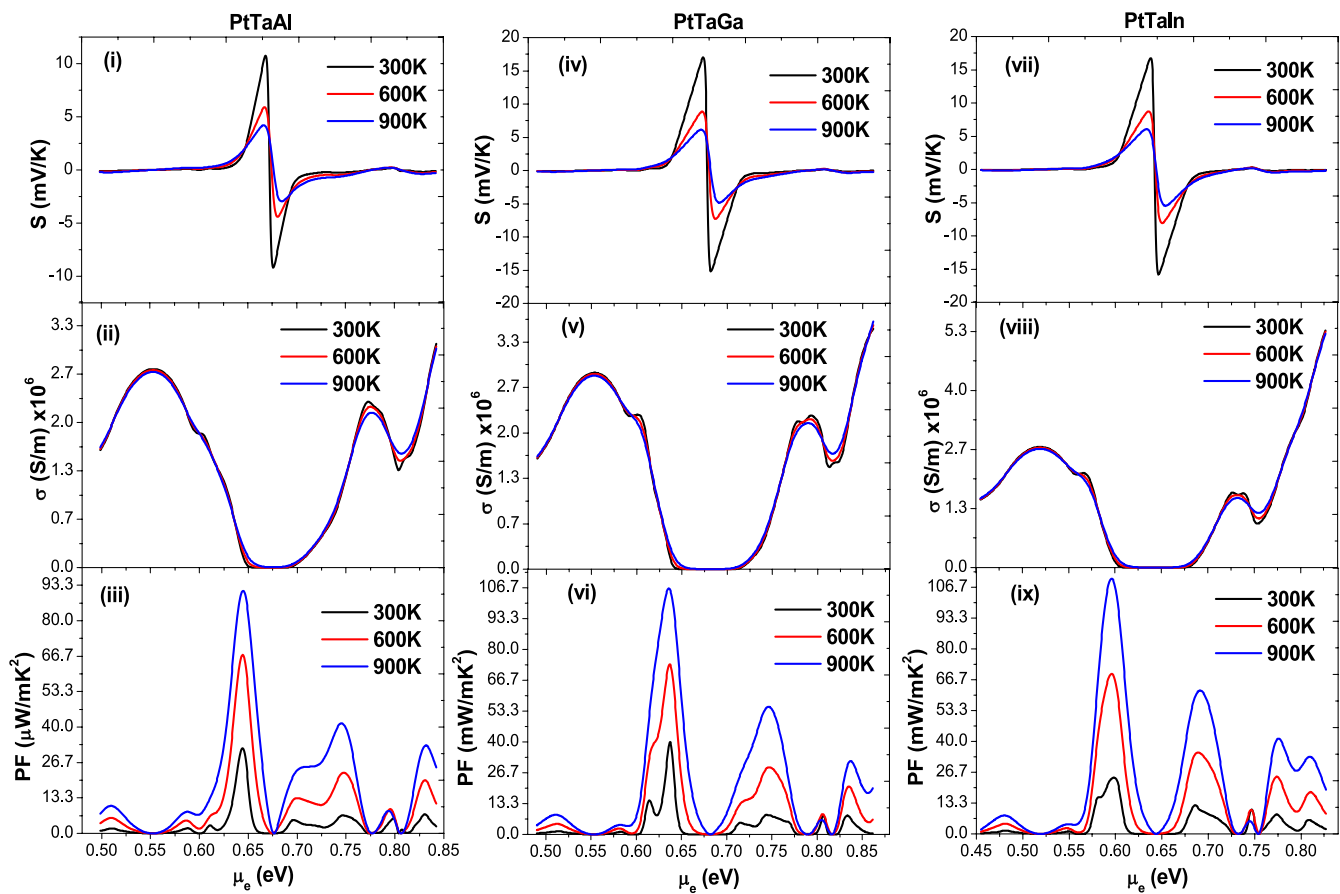

Figure 6. Transport coefficients of $\operatorname{PtTaX}(X=A l, G a, I n)$ alloys as a function of chemical potential: (i, iv, vii) Seebeck coefficient, (iii, v, viii) Electrical conductivity and (iii, vi, ix) Powerfactor.

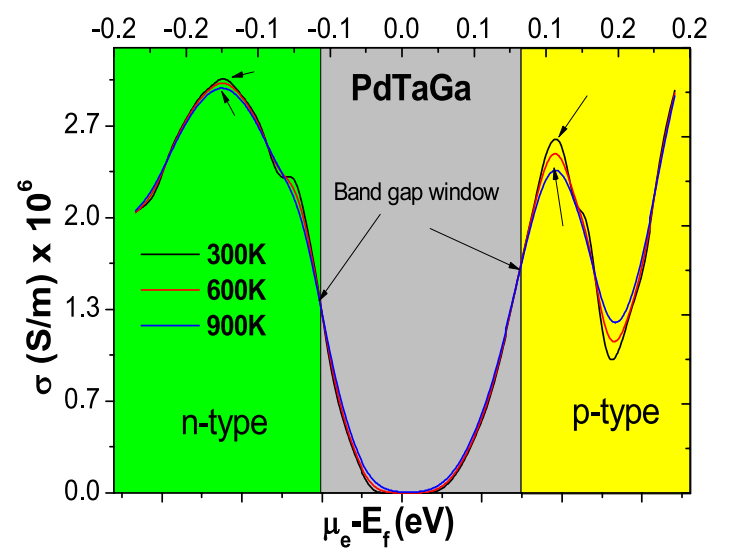

Figure 7. Electrical conductivity of $\mathrm{PdTaGa}(\sigma)$ as a function of chemical potential.

at which $|\mathrm{S}| \sim 1.5-6.0 \mathrm{mV} / \mathrm{K}$. In addition, comparable to the best available TE Heusler $\mathrm{Fe}_{2} \mathrm{Val}\left(4-6 \mathrm{~mW} / \mathrm{mK}^{2}\right)^{59}$, the present alloys then display large $S$ at higher temperatures. Thus, in XTaZ compounds, the improved TE properties at ideal doping concentrations are expected instead by electrons from the transition metal d-eg lowestconduction states and are possibly anticipated in finding applications in high temperature TE materials.

Lattice thermal conductivity. Lattice thermal conductivity is a prerequisite property decided by the phonon spectrum and the phonon-scattering types and rates, their temperature or frequency dependencies, Gruneisen parameters, etc. ${ }^{33,60,61}$. Focusing on the intrinsic thermal conductivity only particular to a specific temperature range, we suppose anharmonic Umklapp (resistive phonon-phonon scattering where the total crystal momentum is not conserved) processes are very important to govern the interactions among the phonons. There occurs another non-resistive phonon scattering process where the conservation of total crystal momentum is observed known as the normal or N-process. This consequences the indirect contribution to the thermal resistance by N-process, although they effectively transfer energy between different phonon modes. Since, Slackmodel mainly focuses on high temperature; $\mathrm{N}$-process occurring frequently at lower temperatures are ignored. Because at low temperatures, N-process is compensated by mass fluctuation and strain field terms in Heusler alloys $^{62}$. Thus, the typical dominance of U-process at higher temperatures when scattering enabled by energy occurs beyond the first Brillouin zone. Therefore, we can say the bulk acoustic phonons are the dominant heat 

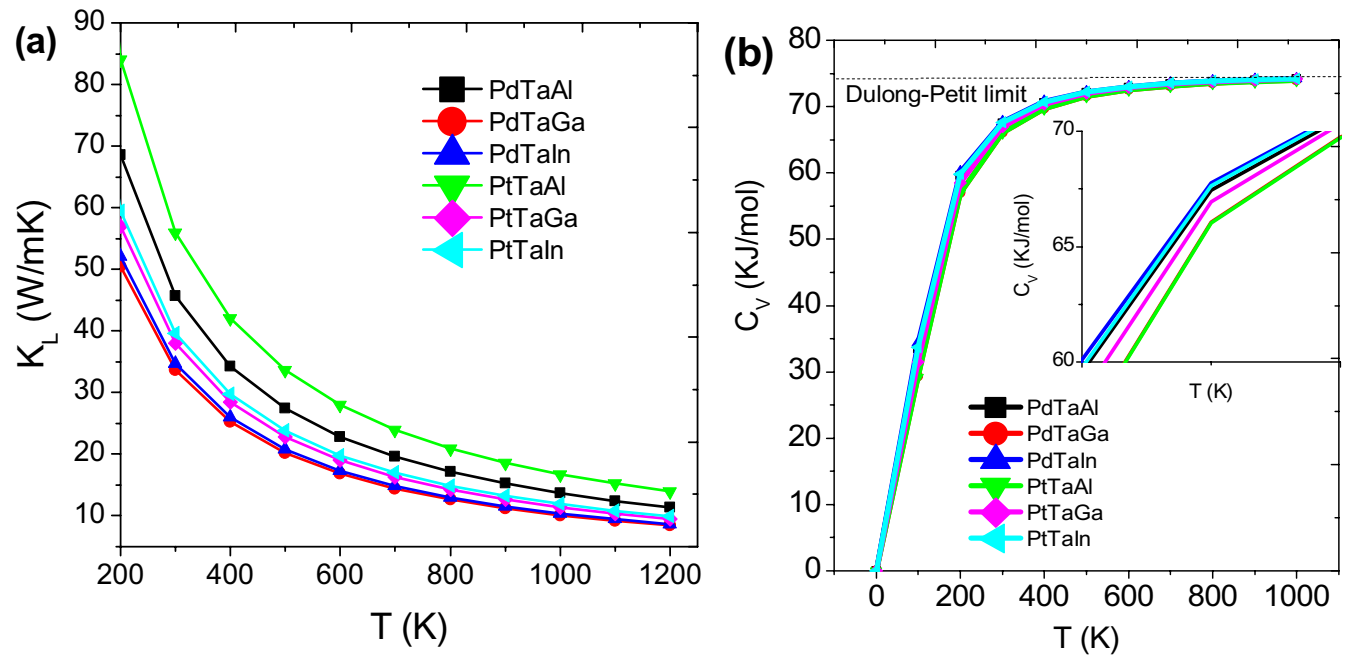

Figure 8. (a) Lattice thermal conductivity $\left(\kappa_{L}\right)$ and (b) Specific heat capacities of XTaZ compounds as a function of temperature.

carriers in this context. This can be observed from the comparative analysis of XTaZ alloys in Fig. 8a, where PtTaAl $\left(147.62 \mathrm{~cm}^{-1}\right)$ and PdTaAl $\left(151.80 \mathrm{~cm}^{-1}\right)$ with higher values of acoustic phonon frequencies are having large thermal conductivity values in comparison to the PdTaGa $\left(135.24 \mathrm{~cm}^{-1}\right)$ and $\mathrm{PtTaGa}\left(143.52 \mathrm{~cm}^{-1}\right)$, respectively. The room temperature values of XTaZ alloys are $45.6 \mathrm{~W} / \mathrm{K}(\mathrm{PdTaAl}), 33.6 \mathrm{~W} / \mathrm{K}(\mathrm{PdTaGa}), 34.6 \mathrm{~W} / \mathrm{K}$ (PdTaIn), 56.1 W/K (PtTaAl), 38.0 W/K (PtTaGa), 39.6 W/K (PtTaIn), respectively. However, these values are quite large in comparison to available thermoelectric materials. For example, $\kappa_{\mathrm{L}}$ for NiTiSn is $9.3 \mathrm{~W} / \mathrm{mK}$ at $300 \mathrm{~K}^{33}$ and for $\mathrm{Fe}_{2} \mathrm{Val}$ is $=28 \mathrm{~W} / \mathrm{mK}$ at $300 \mathrm{~K}^{59}$. Recently, Feng et al.reported PtLaSb $(0.84 \mathrm{~W} / \mathrm{mK}), \mathrm{RhLaTe}$ $(1.21 \mathrm{~W} / \mathrm{mK})$ as the minimum and IrSnTa $(78.09 \mathrm{~W} / \mathrm{mK})$ as the maximum thermal conductivity materials among 74 half-Heusler alloys ${ }^{60}$. Thus, to further the research in having possible TE applications, lattice thermal conductivity needs to be reduced in such alloys and could possibly be achieved via 2D approaches or alloying/ doping $^{63}$. It is well established from experiments and theoretical observations that including vacancy-antisite scattering, grain-boundary scattering, etc. ${ }^{61}$. More importantly, the avoided crossings of the optical phonon and longitudinal acoustic branches are a characteristic of rattling in these alloys. For low thermal conductivity materials, the low frequency phonons are scattered at faster rates. Thus, anharmonic scattering rates, (anharmonicity and the scattering phase space) are clearly significant for thermal conductivity.

Specific heat capacity. Specific heat measurements for XTaZ alloys w.r.t high temperature is entertained by using the quasi-harmonic Debye model ${ }^{49,51,64}$. First, the different total energy versus volume data sets are fitted in static approximation with equation of state (EOS) to predict the crystal parameters at ambient conditions $(\mathrm{P}=0$ and $\mathrm{T}=0)$. Later, the standard thermodynamic relations are utilized to obtain the macroscopic characteristics as a function of temperature. In Fig. 8b, the specific heat capacities of the alloys under study are plotted as a function of temperature. At low temperatures, $\mathrm{C}_{\mathrm{V}}$ is independent of $\mathrm{T}$ and therefore increases until it reaches the Dulong-Petit limit ${ }^{65}$. The unavailability of experimental values of $\mathrm{C}_{\mathrm{V}}$ bars us to compare the data and hence, the approximate Dulong-Petit limit is referenced to be around $75 \mathrm{~kJ} / \mathrm{mol}$.

\section{Conclusion}

To conclude, the structural, mechanical, spintronic properties and phonon dynamics of new $\mathrm{HH}$ materials has been successfully investigated in this report. $F-43 m$ structural symmetry is adopted by these alloys and is dynamically and energetically favored configurations. Electronic structure reveals the semi-conductive nature with energy gaps in the visible range. Elastic calculations describe the mechanical stability of the presumed structures as well as ductile nature. Detailed analysis of material properties in this report pave the way to design novel materials with desired properties experimentally. In the end, the mechanical stability as well as ductile nature is determined, where in the Debye temperature of all these compounds is observed to be within the room temperature range. Our predictions will stimulate extra efforts in experimental and theoretical domains to investigate the possible thermoelectric applications of $\mathrm{HH}$ alloys.

Received: 23 June 2021; Accepted: 11 October 2021

Published online: 21 October 2021

\section{References}

1. Wambach, M. et al. Unraveling self-doping effects in thermoelectric tinisn half-Heusler compounds by combined theory and high-throughput experiments. Adv. Electron. Mater. 2, 1500208 (2016). 
2. Bhattacharya, S. \& Madsen, G. K. H. A novel P-type half-Heusler from high-throughput transport and defect calculations. J. Mater. Chem. C 4, 11261 (2016).

3. Feng, Z., Fu, Y., Putatunda, A., Zhang, Y. \& Singh, D. J. Electronic structure as a guide in screening for potential thermoelectrics: demonstration for half-Heusler compounds. Phys. Rev. B 100, 85202 (2019).

4. Khandy, S. A. \& Da Chai, J. Origin of pseudo gap and thermoelectric signatures of semimetallic Ru2TaGa: structural stability from phonon dynamics, mechanical, and thermodynamic predictions. J. Phys. Chem. Solids 154, 110098 (2021).

5. Xiao, H. et al. Superconductivity in the half-Heusler compound TbPdBi. Phys. Rev. B 97, 224511 (2018).

6. Khandy, S. A. \& Chai, J.-D. Robust stability, half-metallic ferrimagnetism and thermoelectric properties of new quaternary Heusler material: a first principles approach. J. Magn. Magn. Mater. 502, 166562 (2020).

7. Chadov, S., Wu, S.-C., Felser, C. \& Galanakis, I. Stability of Weyl points in magnetic half-metallic Heusler compounds. Phys. Rev. $B$ 96, 024435 (2017).

8. Chang, G. et al. Room-temperature magnetic topological Weyl fermion and nodal line semimetal states in half-metallic Heusler $\mathrm{Co}_{2} \mathrm{TiX}$ (X=Si, Ge, or Sn). Sci. Rep. 6, 38839 (2016).

9. Gao, Q., Opahle, I. \& Zhang, H. High-throughput screening for spin-gapless semiconductors in quaternary Heusler compounds. Phys. Rev. Mater. 3, 024410 (2019).

10. Khandy, S. A. First principles understanding of structural electronic and magnetic properties of new quaternary Heusler alloy: FeVRuSi. Mater. Res. Express 5, 056516 (2018).

11. Saha, R. et al. Intrinsic stability of magnetic anti-skyrmions in the tetragonal inverse Heusler compound $\mathrm{Mn}_{1.4} \mathrm{Pt}_{0.9} \mathrm{Pd}_{0.1} \mathrm{Sn}_{\text {. Nat }}$. Commun. 10, 1-7 (2019).

12. Rana, K. G. et al. Observation of topological hall effect in $\mathrm{Mn}_{2}$ RhSn films. New J. Phys. 18, 085007 (2016).

13. Feng, Y., Cheng, Z. \& Wang, X. Extremely large non-equilibrium tunnel magnetoresistance ratio in CoRhMnGe based magnetic tunnel junction by interface modification. Front. Chem. 7, 550 (2019).

14. Wollmann, L., Nayak, A. K., Parkin, S. S. P. \& Felser, C. Heusler 4.0: tunable materials. Annu. Rev. Mater. Res. 47, 247 (2017).

15. Hinterleitner, B. et al. Thermoelectric performance of a metastable thin-film Heusler alloy. Nature 576, 85 (2019).

16. Cahill, D. G., Watson, S. K. \& Pohl, R. O. Lower limit to the thermal conductivity of disordered crystals. Phys. Rev. B 46, 6131 (1992).

17. Venkatasubramanian, R., Siivola, E., Colpitts, T. \& O'Quinn, B. Thin-film thermoelectric devices with high room-temperature figures of merit. Nature 413, 597-602 (2001).

18. Tan, G. et al. Non-equilibrium processing leads to record high thermoelectric figure of merit in PbTe-SrTe. Nat. Commun. 7, 1-9 (2016).

19. Zhao, L. D. et al. Ultralow thermal conductivity and high thermoelectric figure of merit in SnSe crystals. Nature 508, 373 (2014).

20. Zhu, H. et al. Discovery of TaFeSb-based half-Heuslers with high thermoelectric performance. Nat. Commun. 10, 1-8 (2019).

21. Joshi, G. et al. NbFeSb-based p-type half-Heuslers for power generation applications. Energy Environ. Sci. 7, 4070-4076 (2014).

22. Kaur, K. \& Kumar, R. Ti based half Heusler compounds: a new on the screen with robustic thermoelectric performance. J. Alloys Compd. 727, 1171-1177 (2017).

23. Kaur, K. \& Kumar, R. Giant thermoelectric performance of novel TaIrSn half Heusler compound. Phys. Lett. Sect. A Gen. At. Solid State Phys. 381, 3760 (2017).

24. Feng, L., Liu, E. K., Zhang, W. X., Wang, W. H. \& Wu, G. H. First-principles investigation of half-metallic ferromagnetism of halfHeusler compounds XYZ. J. Magn. Magn. Mater. 351, 92-97 (2014).

25. Dehghan, A. \& Davatolhagh, S. D0-d half-Heusler alloys: a potential class of advanced spintronic materials. J. Alloys Compd. 772, $132-139$ (2019).

26. Wang, X., Cheng, Z. \& Liu, G. Largest magnetic moments in the half-Heusler alloys $\mathrm{XCrZ}(\mathrm{X}=\mathrm{Li}, \mathrm{K}, \mathrm{Rb}, \mathrm{Cs} ; \mathrm{Z}=\mathrm{S}$, Se, Te): a firstprinciples study. Materials (Basel) 10, 1078 (2017).

27. Zahedifar, M. \& Kratzer, P. Band structure and thermoelectric properties of half-Heusler semiconductors from many-body perturbation theory. Phys. Rev. B 97, 035204 (2018).

28. Giannozzi, P. et al. Quantum espresso: a modular and open-source software project for quantum simulations of materials. J. Phys. Condens. Matter 21, 395502 (2009).

29. Perdew, J. P., Burke, K. \& Ernzerhof, M. Generalized gradient approximation made simple. Phys. Rev. Lett. 77, 3865 (1996).

30. Rappe, A. M., Rabe, K. M., Kaxiras, E. \& Joannopoulos, J. D. Optimized pseudopotentials. Phys. Rev. B 41, 1227 (1990).

31. Madsen, G. K. H. \& Singh, D. J. BoltzTraP. A code for calculating band-structure dependent quantities. Comput. Phys. Commun. 175, 67 (2006).

32. Roekeghem, A., Carrete, J., Oses, C., Curtarolo, S. \& Mingo, N. High-throughput computation of thermal conductivity of hightemperature solid phases: the case of oxide and fluoride perovskites. Phys. Rev. X 6, 41061 (2016).

33. Carrete, J., Li, W., Mingo, N., Wang, S. \& Curtarolo, S. Finding unprecedentedly low-thermal-conductivity half-Heusler semiconductors via high-throughput materials modeling. Phys. Rev. X 4, 1 (2014).

34. Malakkal, L., Szpunar, B., Siripurapu, R. K. \& Szpunar, J. A. Thermal conductivity of bulk and nanowire of cubic-SiC from ab initio calculations. Comput. Mater. Sci. 128, 249 (2017).

35. Morelli, D. T. \& Slack, G. A. High lattice thermal conductivity solids. High Therm. Conduct. Mater. 37, 68 (2006).

36. Slack, G. A. Solid State Physics (1979).

37. Hong, A. J. et al. Full-scale computation for all the thermoelectric property parameters of half-Heusler compounds. Sci. Rep. 6, 22778 (2016).

38. Biswas, K. \& Myles, C. W. Electronic and vibrational properties of framework-substituted type-II silicon clathrates. Phys. Rev. B Condens. Matter Mater. Phys. 75, 245205 (2007).

39. Shiomi, J., Esfarjani, K. \& Chen, G. Thermal conductivity of half-Heusler compounds from first-principles calculations. Phys. Rev. B 84, 104302 (2011).

40. Syrbu, N. N., Krasovsky, V. T. \& Grincheshen, I. N. Optical phonons and chemical bonding in TlSbS2, TlSbSe2, and Tl3SbS3 crystals. Cryst. Res. Technol. 28, 371-380 (1993).

41. Loh, E. Covalent bonding effect on optical phonons-empirical relation. Phys. Rev. 169, 750 (1968).

42. Voigt, W. Text Book of Crystal Physics (G.B. Teubner, 1928).

43. Reuss, A. Berechnung Der Fließgrenze von Mischkristallen Auf Grund Der Plastizitätsbedingung Für Einkristalle. ZAMM Z. Angew. Math. Mech. 9, 49-58 (1929).

44. Hill, R. The elastic behaviour of a crystalline aggregate. Proc. Phys. Soc. Sect. A 65, 349 (1952).

45. Ahmad Khandy, S., Kaur, K., Dhiman, S., Singh, J. \& Kumar, V. Exploring thermoelectric properties and stability of half-Heusler PtXSn (X = Zr, Hf) semiconductors: a first principle investigation. Comput. Mater. Sci. 188, 110232 (2021).

46. Shankar, A., Rai, D. P., Khenata, R., Thapa, R. K. \& Mandal, P. K. Energy bands and thermoelectricity of filled skutterudite $\mathrm{EuRu}_{4} \mathrm{As}_{12}$. Int. J. Thermophys. 37, 108 (2016).

47. Kube, C. M. \& De Jong, M. Elastic constants of polycrystals with generally anisotropic crystals. J. Appl. Phys. 120, 165105 (2016).

48. Kaur, K., Rai, D. P., Thapa, R. K. \& Srivastava, S. Structural, electronic, mechanical, and thermoelectric properties of a novel half Heusler compound HfPtPb. J. Appl. Phys. 122, 45110 (2017).

49. Wang, X. et al. Structural, electronic, magnetic, half-metallic, mechanical, and thermodynamic properties of the quaternary Heusler compound FeCrRuSi: a first-principles study. Sci. Rep. 7, 16183 (2017). 
50. Born, M., Huang, K. \& Lax, M. Dynamical theory of crystal lattices. Am. J. Phys. 23, 474 (1955).

51. Khandy, S. A., Islam, I., Gupta, D. C. \& Laref, A. Electronic structure, mechanical and thermodynamic properties of $\mathrm{BaPaO}_{3}$ under pressure. J. Mol. Model. 24, 131 (2018).

52. Wu, S. C., Fecher, G. H., Shahab Naghavi, S. \& Felser, C. Elastic properties and stability of Heusler compounds: cubic Co ${ }_{2}$ YZ compounds with L2 $2_{1}$ structure. J. Appl. Phys. 125, 082523 (2019).

53. Pugh, S. F. Relations between the elastic moduli and the plastic properties of polycrystalline pure metals, London, Edinburgh. Dublin Philos. Mag. J. Sci. 45, 823 (1954).

54. Galanakis, I., Mavropoulos, P. \& Dederichs, P. H. Electronic structure and slater-pauling behaviour in half-metallic Heusler alloys calculated from first principles. J. Phys. D. Appl. Phys. 39, 765 (2006).

55. Xue, Q. Y. et al. LaPtSb: a half-Heusler compound with high thermoelectric performance. Phys. Chem. Chem. Phys. 18, 17912 (2016).

56. Liu, X., Fu, J. \& Chen, G. First-principles calculations of electronic structure and optical and elastic properties of the novel ABX3type LaWN3perovskite structure. RSC Adv. 10, 17317 (2020).

57. Khandy, S. A. \& Gupta, D. C. DFT investigations on mechanical stability, electronic structure and magnetism in Co2TaZ $(\mathrm{Z}=\mathrm{Al}$, Ga, In) Heusler alloys. Semicond. Sci. Technol. 32, 125019 (2017).

58. Bilc, D. I., Hautier, G., Waroquiers, D., Rignanese, G. M. \& Ghosez, P. Low-dimensional transport and large thermoelectric power factors in bulk semiconductors by band engineering of highly directional electronic states. Phys. Rev. Lett. 114, 136601 (2015).

59. Nishino, Y., Deguchi, S. \& Mizutani, U. Thermal and transport properties of the Heusler-type $\mathrm{Fe}_{2} \mathrm{VAl}_{1-\mathrm{x}} \mathrm{Ge}_{\mathrm{x}}(0 \leq \mathrm{x} \leq 0.20)$ alloys: effect of doping on lattice thermal conductivity, electrical resistivity, and seebeck coefficient. Phys. Rev. B Condens. Matter Mater. Phys. 74, 115115 (2006).

60. Feng, Z., Fu, Y., Zhang, Y. \& Singh, D. J. Characterization of rattling in relation to thermal conductivity: ordered half-Heusler semiconductors. Phys. Rev. B 101, 1 (2020).

61. Eliassen, S. N. H. et al. Lattice thermal conductivity of $\mathrm{Ti}_{\mathrm{x}} \mathrm{Zr}_{\mathrm{y}} \mathrm{Hf}_{1-\mathrm{x}-\mathrm{y}} \mathrm{NiSn}$ half-Heusler alloys calculated from first principles: key role of nature of phonon modes. Phys. Rev. B 95, 1 (2017).

62. Petersen, A., Bhattacharya, S., Tritt, T. M. \& Poon, S. J. Critical analysis of lattice thermal conductivity of half-Heusler alloys using variations of callaway model. J. Appl. Phys. 117, 1 (2015).

63. Hooshmand Zaferani, S., Ghomashchi, R. \& Vashaee, D. Strategies for engineering phonon transport in Heusler thermoelectric compounds. Renew. Sustain. Energy Rev. 112, 158 (2019).

64. Blanco, M. A., Francisco, E. \& Luaña, V. GIBBS: isothermal-isobaric thermodynamics of solids from energy curves using a quasiharmonic debye model. Comput. Phys. Commun. 158, 57 (2004).

65. Petit, A. T. \& Dulong, P. L. Study on the measurement of specific heat of solids. Ann. Chim. Phys 10, 395 (1819).

\section{Acknowledgements}

S.A.K. wants to acknowledge National Taiwan University (Grant No. NTU-109L4000), Taiwan for its financial support and Jeng-Da Chai for his useful suggestions. I would like to acknowledge I. Islam, K. Kaur and M.A. Bhat for helping me in solving the comments and reviewing the manuscript.

\section{Author contributions}

S.A.K. designed the project and did the calculations and wrote the manuscript.

\section{Competing interests}

The author declares no competing interests.

\section{Additional information}

Correspondence and requests for materials should be addressed to S.A.K.

Reprints and permissions information is available at www.nature.com/reprints.

Publisher's note Springer Nature remains neutral with regard to jurisdictional claims in published maps and institutional affiliations.

(c) (i) Open Access This article is licensed under a Creative Commons Attribution 4.0 International License, which permits use, sharing, adaptation, distribution and reproduction in any medium or format, as long as you give appropriate credit to the original author(s) and the source, provide a link to the Creative Commons licence, and indicate if changes were made. The images or other third party material in this article are included in the article's Creative Commons licence, unless indicated otherwise in a credit line to the material. If material is not included in the article's Creative Commons licence and your intended use is not permitted by statutory regulation or exceeds the permitted use, you will need to obtain permission directly from the copyright holder. To view a copy of this licence, visit http://creativecommons.org/licenses/by/4.0/.

(C) The Author(s) 2021 